\title{
A Story Coherence based Neural Network Model for Predicting Story Ending
}

\author{
Qian Li*, Ziwei Li, \\ Jin-Mao Wei, Zhenglu Yang* \\ CCCE, Nankai University, China \\ \{liqian515,lzw_nku\}@mail.nankai. \\ edu.cn \\ \{weijm,yangzl\}@nankai.edu.cn
}

\author{
Yanhui $\mathrm{Gu}$ \\ School of CS and Technology, \\ Nanjing Normal University, China \\ gu@njnu.edu.cn
}

\author{
R. Uday Kiran \\ Institute of Industrial Science, \\ The University of Tokyo, Japan \\ uday_rage@tkl.iis.u-tokyo.ac.jp
}

\begin{abstract}
Predicting the ending of a story is an interesting issue that has attracted considerable attention, as in case of the ROC Story Cloze Task (SCT). Although several studies have addressed this issue, the performance remains unsatisfactory due to ineffectiveness of story comprehension. In this paper, we propose to construct a story coherence based neural network model (SCNN) with welldesigned optimizations. The preliminary evaluation demonstrates the effectiveness of our model which is superior to that of state-ofthe-art approaches.
\end{abstract}

\section{Keywords}

natural language processing, neural network, story cloze

\section{Introduction}

Humans have the intrinsic inferential capabilities to predict the development of a story by using commonsense knowledge collected from real life. However, training machines to be able to extract underlying narrative structures to share experiences is an extremely challenging task. Most studies on this subject mainly focus on generating guesses for a missing event or paying attention to specific types of commonsense knowledge.

Different from previous machine comprehension tasks, the Story Cloze Task (SCT) has been proposed as a new evaluation framework to predict what is expected to be the "right" ending to a story plot [5]. The SCT has attracted significant attention. The state-of-the-art mechanical structure involves traditional feature engineering with external knowledge resources, e.g., New York Times data [2], while others introduce neural network-based model to address this issue [1]. However, the performance of this task remains unsatisfactory.

In this paper, we propose an effective story coherence neural network ( $\mathrm{SCNN}$ ) consisting of several optimal components, and we consider features of sentences by adding them as embedding representations. Without the help of external knowledge resources, we obtain the best result $78.3 \%$, which demonstrates that our approach performs better than the state-of-the-art methods.

This paper is published under the Creative Commons Attribution 4.0 International (CC BY 4.0) license. Authors reserve their rights to disseminate the work on their personal and corporate Web sites with the appropriate attribution.

WWW'18 Companion, April 23-27, 2018, Lyon, France

(c) 2018 IW3C2 (International World Wide Web Conference Committee), published under Creative Commons CC BY 4.0 License.

ACM ISBN 978-1-4503-5640-4/18/04.

https://doi.org/10.1145/3184558.3186958

\section{The SCNN Model}

The purpose of this study is to deduce the right ending given its previous context in the story. Formally, given the story $\langle P, E\rangle$, where $P=\left\langle s_{1}, \ldots, s_{n}\right\rangle$ is a story plot, and the ending options $E=\left\langle e_{1}, \ldots, e_{k}\right\rangle$, the task is to select an appropriate ending $e_{i}(1 \leq i \leq k)$ from $E$. We address the task as a regression problem.

The model consists of four layers, as illustrated in Fig. 1. The embedding layer maps each word to a high-dimension vector representation. Then the sentence encoding layer encodes the representation of context, and the feature extraction layer extracts feature from the interaction between plot and ending. Finally, the output layer provide the probability of the right ending.

Embedding Layer: We concatenate five representations: (i) word embedding, (ii) character feature, (iii) part-of-speech (POS) tagging, (iv) sentiment polarity of a word, and (v) negation. Word embedding is conducted by converting token to high-dimensional vector space using 100- $d$ GloVe. The character feature is obtained through a convolutional neural network that outputs a vector for each word by max pooling. By utilizing pre-processing tools, we tackle the last three aspects of a word as one-hot representations, while the POS tagging feature with natural language toolkit, sentiment polarity of a word with a look-up from pre-trained sentiment lexica [2], and negation with a corpus of negation words (i.e., "not", "neither", "nor" and "n't") are collected by ourselves. The concatenation of the five representations of a word is then passed through a two-layer Highway Network to fuse information of features.

Sentence Encoding Layer: We construct a Long Short-Term Memory Network (LSTM) in both directions and concatenate the outputs of forward and backward LSTMs, to learn high level abstractions from time-sequence features of context. We obtain $s_{l j,(j=1, \ldots, n)} \in$ $\mathbb{R}^{2 d \times T}$ for each sentence in plot and $e_{l i} \in \mathbb{R}^{2 d \times G}$ for ending.

The new representations are passed into self-weight operation to model the temporal interactions between words. Taking vector $\mathrm{V}$ as example, we define this operation $\widetilde{V}$ as follows:

$$
M_{i j}=\alpha\left(V_{i}, V_{j}\right) ; \quad a_{i}=\operatorname{softmax}\left(M_{i:}\right) ; \quad \widetilde{V}_{i}=\sum a_{i j} V_{j}
$$

where $\alpha\left(V_{i}, V_{j}\right)=W^{T}\left[V_{i} ; V_{j} ; V_{i} \circ V_{j}\right], W^{T} \in \mathbb{R}^{3 d^{j}}$ is a weight matrix and $\circ$ is element-wise multiplication. The higher-level semantics can directly tackled from encoding sequences through this mechanism. The encodings of story plot is concatenated as $\hat{P}=\left[\widetilde{s_{l 1}} ; \cdots ; \widetilde{s_{l n}}\right]$, while the ending is simply represented as $\hat{e}=\widetilde{e_{l i}}$. 


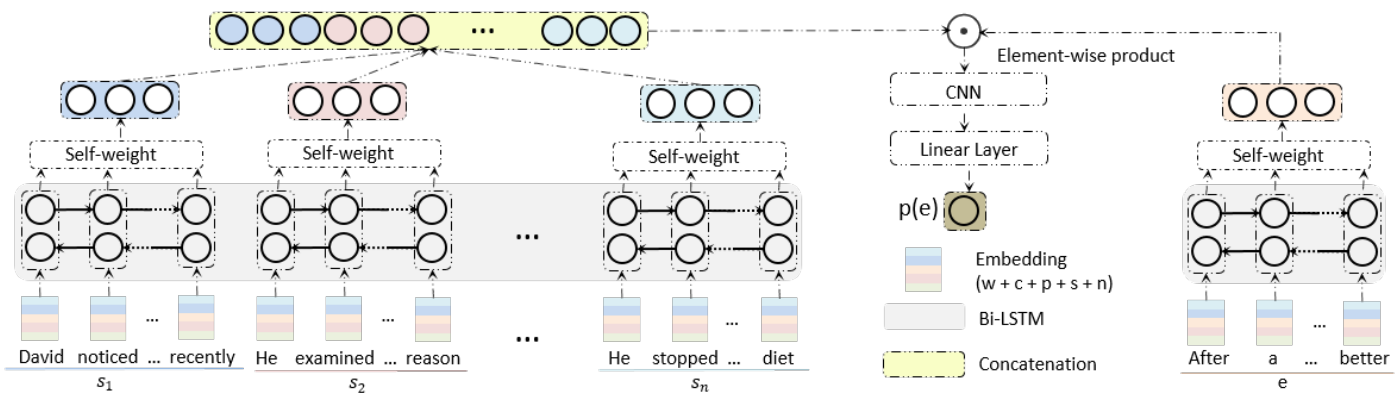

Figure 1: Architecture of $\mathrm{SCNN}^{1}$

Feature Extraction Layer: This layer is inspired by the IIN model [3]. We combine the plot vectors and ending representations with element-wise multiplication to create a word-by-word interaction tensor, in which each channel represents the interaction of the word in one dimension. Then, we adapt DenseNet as a feature extractor that strengthens feature propagation and reduces information disappearance through time.

Output Layer: A linear layer is used to calculate a score to support the prediction.

\section{Experimental Evaluation}

We evaluate our model by using the benchmark SCT Task [5] Notably, the training set contains four-sentence articles with one correct ending, while the evaluation set consists of four-sentence stories with two ending options. The previous studies discarded the training stories and illustrated that learning on the training set was not as satisfactory as it was on the evaluation set $[1,2,6]$. We follow the same strategy used by these state-of-the-art works.

The parameters are optimized by conducting five-fold cross validation and choosing appropriate values based on average held-out accuracy. We use mean-square error (MSE) as the loss function.

The experimental results obtained by comparing our model with state-of-the-art approaches are presented in Table 1. Schwartz [6] is the champion of the LSDSem 2017 Shared Task, which achieves $75.2 \%$ by training a linear regression. HCM [2] trains a joint model with feature engineering that employs external knowledge resources, thereby resulting in $77.6 \%$ precision. The previous NN-based models do not perform well, e.g., Cai [1] obtains $74.7 \%$ accuracy with a hierarchical LSTM and ending2sentence attention. Under the condition in which external resources are not used and the fourth sentence of the plot with ending option are input, SCNN performs much better, i.e., up to $3 \%$ than the state-of-the-art ones. Even compared with the method using external resources, our model achieves better results. SCNN is superior because we deliberately introduce optimal strategies such as concatenated features with embedding representations, embedded into the hierarchical neural network.

We also explore the ablation study [3] on the proposed model to evaluate the effectiveness of each feature and component involved, as illustrated in Fig. 2, showing that character of features and biLSTM of components contribute mainly to our model.

\footnotetext{
${ }^{1} \mathrm{w}$ for word, $\mathrm{c}$ for character, $\mathrm{p}$ for POS, $\mathrm{s}$ for sentiment polarity, $\mathrm{n}$ for negation
}

\begin{tabular}{|ccc|ccc|}
\hline \multicolumn{2}{|c|}{ Machine Learning Algorithm } & \multicolumn{3}{c|}{ Neural Network Algorithm } \\
\hline DSSM[5] & Schwartz[6] & HCM[2] & LSTM[4] & Cai[1] & SCNN \\
\hline $58.5 \%$ & $75.2 \%$ & $77.6 \%$ & $72.8 \%$ & $74.7 \%$ & $\mathbf{7 8 . 3 \%}$ \\
\hline
\end{tabular}

Table 1: Performance comparison on precision

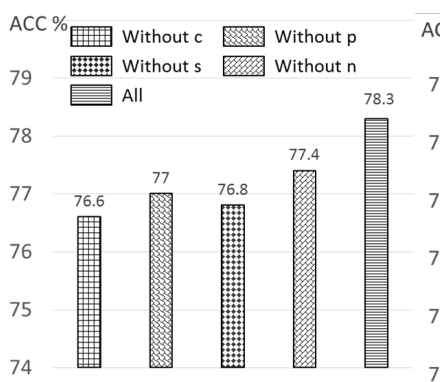

(a) Feature ablation

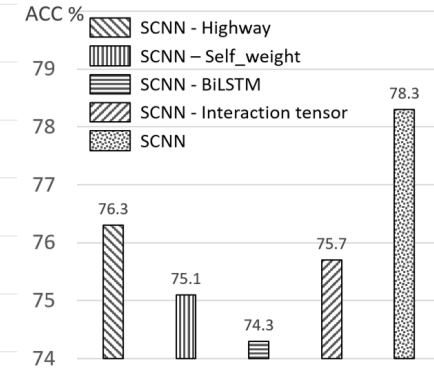

(b) Component ablation
Figure 2: Ablation studies.

\section{Conclusion}

We have introduced an effective story coherence neural network for the Story Cloze Task, with several strategies proposed. The evaluation has demonstrated the effectiveness of our model.

\section{Acknowledgments}

This work was supported in part by the National Natural Science Foundation of China under Grant No.U1636116, 11431006, 61772288, 41571382, the Research Fund for International Young Scientists under Grant No. 61650110510 and 61750110530, and Jiangsu Higher Education Institutions of China under Grant No. 15KJA420001.

\section{References}

[1] Zheng Cai, Lifu Tu, and Kevin Gimpel. 2017. Pay Attention to the Ending:Strong Neural Baselines for the ROC Story Cloze Task. In ACL.

[2] Snigdha Chaturvedi, Haoruo Peng, and Dan Roth. 2017. Story Comprehension for Predicting What Happens Next. In EMNLP.

[3] Yichen Gong, Heng Luo, and Jian Zhang. 2018. Natural language inference over interaction space. In Proc. of ICLR (2018).

[4] Todor Mihaylov and Anette Frank. 2017. Story Cloze Ending Selection Baselines and Data Examination. In LSDSem.

[5] Nasrin Mostafazadeh, Nathanael Chambers, Xiaodong He, Devi Parikh, Dhruv Batra, Lucy Vanderwende, Pushmeet Kohli, and James Allen. 2016. A corpus and evaluation framework for deeper understanding of commonsense stories. In NAACL.

[6] Roy Schwartz, Maarten Sap, Ioannis Konstas, Leila Zilles, Yejin Choi, and Noah A Smith. 2017. The Effect of Different Writing Tasks on Linguistic Style: A Case Study of the ROC Story Cloze Task. In CoNLL. 